\title{
Abel transforms of positive linear operators on weighted spaces
}

\author{
Mehmet Ünver
}

\begin{abstract}
The classical Korovkin approximation theory deals with the convergence of a sequence of positive linear operators. When the sequence of positive linear operators does not converge it will be useful to use some summability methods. In this paper we use the Abel method, a sequence-to-function transformation, to study a Korovkin type approximation theorem for positive linear operators acting from a weighted space $C_{\rho_{1}}$ into a weighted space $B_{\rho_{2}}$. Moreover using the modulus of continuity we also give rate of Abel convergence.
\end{abstract}

\section{Introduction}

The Korovkin theorem provides a criterion for whether a given sequence $\left\{L_{n}\right\}$ of positive linear operators on $C[0,1]$ converges to the identity operator $([2],[12])$. Some variations of this result may be found in [13], [15], [18]. If the sequence of positive linear operators does not converge to the identity operator then it might be beneficial to use some summability methods ([1], [8], [14], [17]). Using the Abel convergence method, recently Unver [19] has studied a Korovkin type approximation theorem for the positive linear operators over the space of continuous functions defined on a closed bounded interval. The purpose of this paper is to use the Abel method, a sequence-to-function transformation, to study a Korovkin type approximation of a function $f$ by means of a sequence $\left\{L_{n}(f ; x)\right\}$

Received by the editors in January 2014 - In revised form in March 2014.

Communicated by F. Bastin.

2010 Mathematics Subject Classification : 41A25, 41A36, 40A05.

Key words and phrases : Abel convergence, sequence of positive linear operators, the Korovkin approximation theorem, weight function, weighted space. 
of positive linear operators acting from a weighted space $C_{\rho_{1}}$ into a weighted space $B_{\rho_{2}}$.

First of all, we give some basic definitions and notations used in this paper:

A real valued function $\rho$ is called a weight function if it is continuous on $\mathbb{R}$, $\rho(x) \geq 1$ for all $x \in \mathbb{R}$ and

$$
\lim _{|x| \rightarrow \infty} \rho(x)=\infty
$$

where $\mathbb{R}$ denotes the set of all real numbers.

Let $\rho$ be a weight function. The space of real valued functions $f$ defined on $\mathbb{R}$ and for all $x \in \mathbb{R}$ satisfying $|f(x)| \leq K_{f} \rho(x)$ is called the weighted space and denoted by $B_{\rho}$, where $K_{f}$ is a constant depending on $f$. The weighted subspace $C_{\rho}$ of $B_{\rho}$ is given by

$$
\left\{C_{\rho}:=f \in B_{\rho}: f \text { is continuous on } \mathbb{R}\right\} .
$$

It is known [9] that the spaces $B_{\rho}$ and $C_{\rho}$ are Banach spaces with the norm

$$
\|f\|_{\rho}:=\sup _{x \in \mathbb{R}} \frac{|f(x)|}{\rho(x)} .
$$

Let $L: C_{\rho_{1}} \rightarrow B_{\rho_{2}}$ be a linear operator. Then $L$ is called positive if $L f \geq 0$ whenever $f \geq 0$. If $L$ is a positive linear operator then $f \leq g$ implies that $L f \leq L g$ and, $|f| \leq g$ implies $|L f| \leq L g$.

The following approximation theorem for a sequence of positive linear operators acting from $C_{\rho_{1}}$ into $B_{\rho_{2}}$ may be found in [9] and [10].

Theorem A. Assume that $\rho_{1}$ and $\rho_{2}$ are weight functions such that

$$
\lim _{|x| \rightarrow \infty} \frac{\rho_{1}(x)}{\rho_{2}(x)}=0
$$

and $\left\{L_{n}\right\}$ is a sequence of positive linear operators from $C_{\rho_{1}}$ into $B_{\rho_{2}}$. Then $\lim _{n}\left\|L_{n} f-f\right\|_{\rho_{2}}=0$ for every $f \in C_{\rho_{1}}$ if and only if $\lim _{n}\left\|L_{n} F_{i}-F_{i}\right\|_{\rho_{1}}=0$ for $i=0,1,2$ where

$$
F_{i}=\frac{x^{i} \rho_{1}(x)}{1+x^{2}}, i=0,1,2
$$

Some analogs of this theorem can be found in [3], [4] and [7].

In the present paper, using the Abel method, we will give another analog of Theorem A.

Let us recall the Abel method:

If the series

$$
\sum_{k=0}^{\infty} x_{k} \alpha^{k}
$$

converges for all $\alpha \in(0,1)$ and

$$
\lim _{\alpha \rightarrow 1^{-}}(1-\alpha) \sum_{k=0}^{\infty} x_{k} \alpha^{k}=L
$$

then we say that the sequence $x=\left(x_{k}\right)$ is Abel convergent to $L$. 


$$
\begin{aligned}
& \text { As } \frac{1}{1-\alpha}=\sum_{k=0}^{\infty} \alpha^{k}, 0<\alpha<1,(1.2) \text { is equivalent to the following: } \\
& \qquad \lim _{\alpha \rightarrow 1^{-}}(1-\alpha) \sum_{k=0}^{\infty}\left(x_{k}-L\right) \alpha^{k}=0 .
\end{aligned}
$$

Note that the convergence of a sequence implies the Abel convergence of it, but not conversely ([5], [16]).

Let $\left\{L_{n}\right\}$ be a sequence of positive linear operators from $C_{\rho_{1}}$ into $B_{\rho_{2}}$ such that

$$
\sum_{n=0}^{\infty}\left\|L_{n}\left(\rho_{1}\right)\right\|_{\rho_{2}} \alpha^{n}<\infty
$$

for all $\alpha \in(0,1)$, then for all $f \in C_{\rho_{1}}$ the series $\sum_{n=0}^{\infty} L_{n}(f(t) ; x) \alpha^{n}$ converges. Hence the operator $U_{\alpha}$ defined by

$$
U_{\alpha}(f ; x):=(1-\alpha) \sum_{n=0}^{\infty} L_{n}(f(t) ; x) \alpha^{n}
$$

is a positive linear operator from $C_{\rho_{1}}$ to $B_{\rho_{2}}$ which is bounded for all $\alpha \in(0,1)$. Thus

$$
\begin{aligned}
\left\|U_{\alpha}\right\|_{C_{\rho_{1}} \rightarrow B_{\rho_{2}}} & =\sup _{\|f\|_{\rho_{1}}=1}\left\|U_{\alpha} f\right\|_{\rho_{2}} \\
& \leq \sup _{x \in \mathbb{R}} \frac{(1-\alpha)\left|\sum_{n=0}^{\infty} L_{n}\left(\rho_{1} ; x\right) \alpha^{n}\right|}{\rho_{2}} \\
& =\left\|U_{\alpha} \rho_{1}\right\|_{\rho_{2}}
\end{aligned}
$$

for all $\alpha \in(0,1)$.

\section{Approximation by Abel Method on Weighted Spaces}

In this section using the Abel method we study a Korovkin type approximation theorem for a sequence of positive linear operators acting from $C_{\rho_{1}}$ into $B_{\rho_{2}}$.

We need the following lemmas.

Lemma 1. Let $\left\{L_{n}\right\}$ be a sequence of positive linear operators from $C_{\rho_{1}}$ into $B_{\rho_{2}}$ such that (1.3) holds and let $\rho_{1}$ and $\rho_{2}$ be weight functions satisfying (1.1). Assume that

$$
\sup _{\alpha \in(0,1)}\left\|U_{\alpha}\right\|_{C_{\rho_{1}} \rightarrow B_{\rho_{1}}}<\infty
$$

If for any $s \in \mathbb{R}$,

$$
\lim _{\alpha \rightarrow 1^{-}} \sup _{\|f\|_{\rho_{1}}=1} \sup _{|x| \leq s} \frac{\left|U_{\alpha}(f ; x)\right|}{\rho_{1}(x)}=0
$$


then

$$
\lim _{\alpha \rightarrow 1^{-}}\left\|U_{\alpha}\right\|_{C_{\rho_{1}} \rightarrow B_{\rho_{2}}}=0
$$

Proof. The proof can be obtained easily by applying the same arguments used in Lemma 2 [10]. But we include the proof for the sake of completeness.

It follows from (1.1) that, for any $\varepsilon>0$, there exists a number $s_{0}$ such that $\rho_{1}(x) \leq$ $\varepsilon \rho_{2}(x)$ for all $|x|>s_{0}$. By the continuity of $\rho_{1} / \rho_{2}$, there exists $K>0$ such that $\rho_{1}(x) \leq K \rho_{2}(x)$ for all $|x| \leq s_{0}$. Hence we get

$$
\begin{aligned}
\left\|U_{\alpha}\right\|_{C_{\rho_{1}} \rightarrow B_{\rho_{2}}} & =\sup _{\|f\|_{\rho_{1}}=1}\left\|U_{\alpha} f\right\|_{\rho_{2}} \\
& =\sup _{\|f\|_{\rho_{1}}=1} \sup _{x \in \mathbb{R}} \frac{\left|U_{\alpha}(f ; x)\right|}{\rho_{2}} \\
& \leq \sup _{\|f\|_{\rho_{1}}=1|x| \leq s_{0}} \frac{\left|U_{\alpha}(f ; x)\right|}{\rho_{2}} \\
& +\sup _{\|f\|_{\rho_{1}}=1|x|>s_{0}} \frac{\left|U_{\alpha}(f ; x)\right|}{\rho_{2}} \\
& \leq K \sup _{\|f\|_{\rho_{1}}=1|x| \leq s_{0}} \frac{\left|U_{\alpha}(f ; x)\right|}{\rho_{1}}+\varepsilon\left\|U_{\alpha}\right\|_{C_{\rho_{1}} \rightarrow B_{\rho_{1}}} .
\end{aligned}
$$

Then from (2.1) and (2.2) we have

$$
\lim _{\alpha \rightarrow 1^{-}}\left\|U_{\alpha}\right\|_{C_{\rho_{1}} \rightarrow B_{\rho_{2}}}=0
$$

which concludes the proof.

Lemma 2. Let $\left\{L_{n}\right\}$ be a sequence of linear operators from $C_{\rho_{1}}$ into $B_{\rho_{2}}$ such that (1.3), (1.1) and (2.1) hold. If for any $s \in \mathbb{R}$,

$$
\lim _{\alpha \rightarrow 1^{-}} \sup _{\|f\|_{\rho_{1}}=1} \sup _{|x| \leq s}\left|U_{\alpha}(f ; x)-f(x)\right|=0
$$

then

$$
\lim _{\alpha \rightarrow 1^{-}}\left\|U_{\alpha} f-f(x)\right\|_{\rho_{2}}=0
$$

for any $f \in C_{\rho_{1}}$.

Proof. Let $I$ be the identity operator on $C_{\rho_{1}}$. Let $T_{n}:=L_{n}-I$ and consider the operator $V_{\alpha}$ defined by

$$
V_{\alpha}(f ; x)=(1-\alpha) \sum_{n=0}^{\infty} T_{n}(f(t) ; x) \alpha^{n}
$$


for all $\alpha \in(0,1)$, which is well defined from (1.3) and belongs to $B_{\rho_{2}}$. Since

$$
\begin{aligned}
\left\|V_{\alpha}\right\|_{C_{\rho_{1}} \rightarrow B_{\rho_{1}}} & =\sup _{\|f\|_{\rho_{1}}=1}\left\|V_{\alpha} f\right\|_{\rho_{1}} \\
& =\sup _{\|f\|_{\rho_{1}}=1} \sup _{x \in \mathbb{R}} \frac{\left|V_{\alpha}(f ; x)\right|}{\rho_{1}(x)} \\
& \leq \sup _{\|f\|_{\rho_{1}}=1} \sup _{x \in \mathbb{R}} \frac{\left|U_{\alpha}(f ; x)\right|}{\rho_{1}(x)} \\
& +\sup _{\|f\|_{\rho_{1}}=1} \sup _{x \in \mathbb{R}}(1-\alpha) \frac{\left|\sum_{n=0}^{\infty} f(x) \alpha^{n}\right|}{\rho_{1}(x)} \\
& =\left\|U_{\alpha}\right\|_{C_{\rho_{1}} \rightarrow B_{\rho 1}}+\sup _{\|f\|_{\rho_{1}}=1}\|f\|_{\rho_{1}}(1-\alpha) \sum_{n=0}^{\infty} \alpha^{n} \\
& =\left\|U_{\alpha}\right\|_{C_{\rho_{1}} \rightarrow B_{\rho 1}}+1
\end{aligned}
$$

it follows from (2.1) that

$$
\sup _{\alpha \in(0,1)}\left\|V_{\alpha}\right\|_{C_{\rho_{1}} \rightarrow B_{\rho_{1}}}<\infty
$$

As $\rho_{1}>1$ we have for any $s \in \mathbb{R}$ that

$$
\begin{aligned}
\sup _{\|f\|_{\rho_{1}}=1|x| \leq s} \sup _{\mid} \frac{\left|V_{\alpha}(f ; x)\right|}{\rho_{1}(x)} & =\sup _{\|f\|_{\rho_{1}}=1|x| \leq s} \frac{\left|U_{\alpha}(f ; x)-f(x)\right|}{\rho_{1}(x)} \\
& \leq \sup _{\|f\|_{\rho_{1}}=1|x| \leq s} \sup _{|x| \leq s}\left|U_{\alpha}(f ; x)-f(x)\right|
\end{aligned}
$$

Then from (2.3) and (2.4) we have for any $s \in \mathbb{R}$ that

$$
\lim _{\alpha \rightarrow 1^{-}} \sup _{\|f\|_{\rho_{1}}=1} \sup _{|x| \leq s} \frac{\left|V_{\alpha}(f ; x)\right|}{\rho_{1}(x)}=0
$$

Hence it follows from Lemma 1 that

$$
\lim _{\alpha \rightarrow 1^{-}}\left\|V_{\alpha}\right\|_{C_{\rho_{1}} \rightarrow B_{\rho_{2}}}=0
$$


Now we get for any $f \in C_{\rho_{1}}$ that

$$
\begin{aligned}
\left\|U_{\alpha} f-f\right\|_{\rho_{2}} & =\sup _{x \in \mathbb{R}} \frac{\left|(1-\alpha) \sum_{n=0}^{\infty} L_{n}(f ; x) \alpha^{n}-f(x)\right|}{\rho_{2}(x)} \\
& =\sup _{x \in \mathbb{R}} \frac{\left|(1-\alpha) \sum_{n=0}^{\infty}\left(L_{n}(f ; x)-f(x)\right) \alpha^{n}\right|}{\rho_{2}(x)} \\
& =\sup _{x \in \mathbb{R}} \frac{\left|(1-\alpha) \sum_{n=0}^{\infty} T_{n}(f ; x) \alpha^{n}\right|}{\rho_{2}(x)} \\
& \leq\left\|V_{\alpha} f\right\|_{\rho_{2}} \\
& \leq\left\|V_{\alpha}\right\|_{C_{\rho_{1}} \rightarrow B_{\rho_{2}}}\|f\|_{\rho_{1}}
\end{aligned}
$$

Hence by (2.5) we have

$$
\lim _{\alpha \rightarrow 1^{-}}\left\|U_{\alpha} f-f\right\|_{\rho_{2}}=0
$$

Now we are ready to give our main result.

Theorem 1. Let $\left\{L_{n}\right\}$ be a sequence of positive linear operators from $C_{\rho_{1}}$ into $B_{\rho_{2}}$ such that (1.1) and (1.3) hold. If

$$
\lim _{\alpha \rightarrow 1^{-}}\left\|U_{\alpha} F_{i}-F_{i}\right\|_{\rho_{1}}=0
$$

then for all $f \in C_{\rho_{1}}$

$$
\lim _{\alpha \rightarrow 1^{-}}\left\|U_{\alpha} f-f\right\|_{\rho_{2}}=0
$$

where $F_{i}(x)=\frac{x^{i} \rho_{1}(x)}{1+x^{2}}, i=0,1,2$.

Proof. Let $f \in C_{\rho_{1}}$ and assume that (2.6) holds. It is obvious that (2.1) holds. Since $f \in C_{\rho_{1}}$ there exists a constant $M_{f}$ such that $|f(x)| \leq M_{f} \rho_{1}(x)$ for all $x \in \mathbb{R}$. By using the same arguments in Theorem 14 of [11], we have for all $\alpha \in(0,1)$ and any $s \in \mathbb{R}$ that

$$
\begin{aligned}
\sup _{\|f\|_{\rho_{1}}=1|x| \leq s} \sup _{|x| \leq s}\left|U_{\alpha}(f ; x)-f(x)\right| & \leq K\left\{\varepsilon\left\|U_{\alpha}\right\|_{C_{\rho_{1}} \rightarrow B_{\rho_{1}}}\right. \\
& +\left\|U_{\alpha} F_{2}-F_{2}\right\|_{\rho_{1}} \\
& +\left\|U_{\alpha} F_{1}-F_{1}\right\|_{\rho_{1}} \\
& \left.+\left\|U_{\alpha} F_{0}-F_{0}\right\|_{\rho_{1}}\right\}
\end{aligned}
$$

where $K:=\max \left\{1+K_{2} K_{3}, C\left(K_{1}+K_{2} K_{4}\right)+K_{2} K_{3}\right\}, K_{1}:=K_{1}(s)=\sup _{|x| \leq s}|f(x)|$, $K_{2}:=K_{2}(s)=\sup _{|x| \leq s} H_{\rho_{1}}(x), K_{3}:=K_{3}(s)=\sup _{|x| \leq s}\left\{\frac{\rho_{1}(x)}{F_{0}(x)}\right\}$, 
$K_{4}:=K_{4}(s)=\sup _{|x| \leq s}\left\{\frac{H_{\rho_{1}}(x)}{F_{0}(x)}\right\}, H_{\rho_{1}}(x)=4 M_{f} \rho_{1}(x)\left\{1+\frac{1+x^{2}}{\delta^{2}}\right\}$ and $C:=\max \left\{\sup _{|x| \leq s} \rho_{1}(x), 2 \sup _{|x| \leq s}|x| \rho_{1}(x), \sup _{|x| \leq s} x^{2} \rho_{1}(x)\right\}$. Hence from (2.1) and (2.6) we have for any $s \in \mathbb{R}$ that

$$
\lim _{\alpha \rightarrow 1^{-}} \sup _{\|f\|_{\rho_{1}}=1} \sup _{|x| \leq s}\left|U_{\alpha}(f ; x)-f(x)\right|=0
$$

Then by Lemma 2 we have

$$
\lim _{\alpha \rightarrow 1^{-}}\left\|U_{\alpha} f-f\right\|_{\rho_{2}}=0
$$

which concludes the proof.

\section{Rate of Abel Convergence}

We consider the following weighted modulus of continuity

$$
\omega_{\rho_{1}}(f, \delta)=\sup _{|t-x| \leq \delta}\left\{\frac{|f(t)-f(x)|}{\rho_{1}(x)}\right\}
$$

where $\delta$ is a positive constant and $f \in C_{\rho_{1}}$. It was shown in [6] that $\omega_{\rho_{1}}(f, \delta)$ is a weighted modulus of continuity and it is well known that, for all $f \in C_{\rho_{1}}$ and for all $c>0$,

$$
\omega_{\rho_{1}}(f, c \delta) \leq(1+[|c|]) \omega_{\rho_{1}}(f, \delta)
$$

where $[|c|]$ is the greatest integer less than or equal to $c$.

In this section, using weighted modulus of continuity, we study the rate of Abel convergence.

Lemma 3. Let $\left\{L_{n}\right\}$ be a sequence of positive linear operators from $C_{\rho_{1}}$ into $B_{\rho_{2}}$ such that (1.3), (1.1) and (2.1) hold and let $L_{n} \varphi_{x}$ and $L_{n} F_{0}$ be in $C_{\rho_{1}}$ for each $n$ where $\varphi_{x}(t):=$ $(t-x)^{2}$ and $F_{0}(t)=1$. Then for any $s>0$ and all $\alpha \in(0,1)$

$$
\begin{aligned}
\sup _{\|f\|_{\rho_{1}}=1|x| \leq s} \sup _{|x| \leq s}\left|U_{\alpha}(f ; x)-f(x)\right| & \leq K\left\{\sup _{\|f\|_{\rho_{1}}=1} \omega_{\rho_{1}}(f, \psi(\alpha))\right. \\
& \left.+\left\|U_{\alpha} F_{0}-F_{0}\right\|_{\rho_{1}}\right\}
\end{aligned}
$$

holds, where $\psi(\alpha)=\sqrt{\left\|U_{\alpha} \varphi_{x}\right\|_{\rho_{1}}}$ and $K:=K(s)$ is a positive constant.

Proof. Using the linearity and positivity of $U_{\alpha}$, for all $\alpha \in(0,1), \delta>0$ and $f \in C_{\rho_{1}}$ 
we have

$$
\begin{aligned}
\left|U_{\alpha}(f ; x)-f(x)\right| & \leq U_{\alpha}\{|f(t)-f(x)| ; x\} \\
& +|f(x)|\left|U_{\alpha}\left(F_{0} ; x\right)-F_{0}(x)\right| \\
& \leq U_{\alpha}\left(\rho_{1}(x) \omega_{\rho_{1}}\left(f, \frac{|t-x|}{\delta} \delta\right) ; x\right) \\
& +|f(x)|\left|U_{\alpha}\left(F_{0} ; x\right)-F_{0}(x)\right| \\
& \leq U_{\alpha}\left(\left(1+\left[\left|\frac{|t-x|}{\delta}\right|\right]\right) \rho_{1}(x) \omega_{\rho_{1}}(f, \delta) ; x\right) \\
& +|f(x)|\left|U_{\alpha}\left(F_{0} ; x\right)-F_{0}(x)\right| \\
& \leq \rho_{1}(x) \omega_{\rho_{1}}(f, \delta) U_{\alpha}\left(1+\frac{(t-x)^{2}}{\delta^{2}} ; x\right) \\
& +|f(x)|\left|U_{\alpha}\left(F_{0} ; x\right)-F_{0}(x)\right| \\
& \leq \rho_{1}(x) \omega_{\rho_{1}}(f, \delta)\left\{U_{\alpha}\left(\rho_{1} ; x\right)\right. \\
& \left.+\frac{1}{\delta^{2}} U\left\{\left(\varphi_{x}(t) ; x\right) ; y\right\}\right\} \\
& +|f(x)|\left|U_{\alpha}\left(F_{0} ; x\right)-F_{0}(x)\right| .
\end{aligned}
$$

Since $\varphi_{x} \in C_{\rho_{1}}$, for any $s>0$ and all $\alpha \in(0,1)$, we get from (3.3) that

$$
\begin{aligned}
\sup _{\|f\|_{\rho_{1}}=1|x| \leq s} \sup _{|x| \leq s}\left|U_{\alpha}(f ; x)-f(x)\right| & \leq K_{1}^{2} \sup _{\|f\|_{\rho_{1}}=1} \omega_{\rho_{1}}(f, \delta)\left\{\left\|U_{\alpha}\right\|_{C_{\rho_{1}} \rightarrow B_{\rho_{1}}}\right. \\
& \left.+\frac{1}{\delta^{2}}\left\|U_{\alpha} \varphi_{x}\right\|_{\rho_{1}}\right\} \\
& +K_{2}\left\|U_{\alpha} F_{0}-F_{0}\right\|_{\rho_{1}}
\end{aligned}
$$

where $K_{1}=\sup _{|x| \leq s} \rho_{1}(x)=1+s^{2}$ and $K_{2}=\sup _{|x| \leq s} \frac{f(x)}{\rho_{1}(x)}$. By the hypotheses, for all $\alpha \in(0,1)$, we have

$$
\left\|U_{\alpha}\right\|_{C_{\rho_{1}} \rightarrow B_{\rho_{1}}} \leq M
$$

Now putting $\delta=\psi(\alpha)=\sqrt{\left\|U_{\alpha} \varphi_{x}\right\|_{\rho_{1}}}$ and by (3.4) we have, for all $\alpha \in(0,1)$, that

$$
\begin{aligned}
\sup _{\|f\|_{\rho_{1}}=1|x| \leq s} \sup _{|x|}\left|U_{\alpha}(f ; x)-f(x)\right| & \leq K\left\{\sup _{\|f\|_{\rho_{1}}=1} \omega_{\rho_{1}}(f, \psi(\alpha))\right. \\
& \left.+\left\|U_{\alpha} F_{0}-F_{0}\right\|_{\rho_{1}}\right\}
\end{aligned}
$$

where $K=\max \left\{K_{1}^{2}(1+M), K_{2}\right\}$.

Theorem 2. Let $\left\{L_{n}\right\}$ be a sequence of positive linear operators from $C_{\rho_{1}}$ into $B_{\rho_{2}}$ such that (1.3), (1.1) and (2.1) hold and let $L_{n} \varphi_{x}$ and $L_{n} F_{0}$ be in $C_{\rho_{1}}$ for each $n$ where $\varphi_{x}(t):=$ $(t-x)^{2}$ and $F_{0}(t)=1$. If 
i) $\lim _{\alpha \rightarrow 1^{-}}\left\|U_{\alpha} F_{0} t-F_{0}\right\|_{\rho_{1}}=0$

ii) $\lim _{\alpha \rightarrow 1^{-}} \sup _{\|f\|_{\rho_{1}}=1} \omega_{\rho_{1}}(f, \psi(\alpha))=0$

then for any $f \in C_{\rho_{1}}$ we get

$$
\lim _{\alpha \rightarrow 1^{-}}\left\|U_{\alpha} f-f\right\|_{\rho_{2}}=0 .
$$

Proof. By (i), (ii) and Lemma 3, we have

$$
\lim _{\alpha \rightarrow 1^{-}} \sup _{\|f\|_{\rho_{1}}=1|x| \leq s} \sup _{\alpha}\left|U_{\alpha}(f ; x)-f(x)\right|=0 .
$$

Then for all $f \in C_{\rho_{1}}$, it follows from Lemma 2 that

$$
\lim _{\alpha \rightarrow 1^{-}}\left\|U_{\alpha} f-f\right\|_{\rho_{2}}=0 .
$$

\section{Remarks}

Let $\rho_{1}$ and $\rho_{2}$ be weight functions satisfying (1.1) and $\left\{T_{n}\right\}$ be a sequence of positive linear operators from $C_{\rho_{1}}$ to $B_{\rho_{2}}$ satisfying the hypotheses of Theorem $A$. Now define a sequence $\alpha=\left(\alpha_{n}\right)$ as $\alpha_{n}=1$ if $n$ is a perfect square, and $\alpha_{n}=0$ otherwise. Note that $\alpha$ is Abel convergent to zero but not convergent. Let $\left\{L_{n}\right\}$ be a sequence of positive linear operators acting from $C_{\rho_{1}}$ into $B_{\rho_{2}}$ defined as

$$
L_{n}(f ; x)=\left(1+\alpha_{n}\right) T_{n}(f)
$$

for $f \in C_{\rho_{1}}$. Observe that the sequence $\left\{L_{n}\right\}$ does not satisfy the hypotheses of Theorem A but it satisfies the hypotheses of our Theorem 1.

\section{References}

[1] O. Agratini, On statistical approximation in spaces of continuous functions, Positivity 13, no. 4, (2009), 735-743;

[2] F. Altomare and M. Campiti, Korovkin type Approximation Theory and its Application, Walter de Gruyter Publ. Berlin, 1994;

[3] Ö. G. Atlihan and C. Orhan, Summation process of positive linear operators, Comput. Math. Appl. 56, no. 5, (2008), 1188-1195;

[4] Ö. G.Atlihan and C. Orhan, Matrix summability and positive linear operators, Positivity 11 , no. 3, (2007) 387-398;

[5] J. Boos, Classical and Modern Methods in Summability, Oxford Univ. Press, 2000;

[6] O. Doğru, Weighted approximation of continuous functions on the all positive axis by modified linear positive operators. Int. J. Comput. Numer. Anal. Appl. 1, no. 2, (2002), 135-147; 
[7] O. Duman and C. Orhan, Statistical approximation by positive linear operators, Studia Math. 161, no. 2, (2004), 187-197;

[8] A. D. Gadjiev and C. Orhan, Some approximation theorems via statistical convergence, Rocky Mountain J. Math. 32, (2002), 129-138;

[9] A. D. Gadjiev, Theorems of the type of P. P. Korovkin's theorems, Mat. Zametki 20, (1976), 781-786 (in Russian);

[10] -, The convergence problem for a sequence of positive linear operators on unbounded sets, and theorems analogous to that of P. P. Korovkin, Soviet Math. Dokl. 15, (1974), 1433-1436;

[11] A. D. Gadjiev and H. H. Hacısalihoğlu, Positive Linear Operators, in Turkish, Ankara University Press, no. 35, 1995;

[12] P. P. Korovkin, Linear Operators and Approximation Theory, Hindustan Publ. Co., Delhi, (1960).

[13] H. E. Lomelí and C. L. García, Variations on a theorem of Korovkin, Amer. Math. Monthly 113, (2006), 744-750;

[14] R. N. Mohapatra, Quantitative results on almost convergence of a sequence of positive linear operators, J. Approximation Theory 20, (1977), 239-250;

[15] T. Nishishiraho, Convergence of positive linear approximation processes, Tohoku Math. J. (2) 35, (1983), 441-458;

[16] R. E. Powell and S. M. Shah, Summability Theory and Applications, Prentice-Hall of India, New Delhi, 1988;

[17] J. J. Swetits, On summability and positive linear operators, J. Approx. Theory 25, (1979) 186-188;

[18] M. Uchiyama, Proofs of Korovkin's theorems via inequalities, Amer. Math. Monthly 110, (2003), 334-336;

[19] M. Unver, Abel transforms of positive linear operators, AIP Conference Proceedings 1558, (2013), 1148-1151.

Ankara University,

Faculty of Science, Department of Mathematics,

Tandogan,

Ankara, TURKEY

email:munver@ankara.edu.tr 